\title{
Ultrasound assessment of muscle mass and quality: it has never been so easy!
}

\author{
Karolina Piotrowicz ${ }^{1}$ (1) Jerzy Gąsowski ${ }^{1}$
}

Received: 3 November 2018 / Accepted: 13 November 2018 / Published online: 30 November 2018

(c) The Author(s) 2018

Sarcopenia is rarely screened for and diagnosed in everyday clinical practice. This is despite the fact that sarcopenia in old age translates into a higher risk of disability and dependence, increased demand for institutionalization and rehabilitation, greater morbidity and mortality, and finally considerable healthcare-related expenditure [1].

In the current issue of the European Geriatric Medicine, Ramirez-Fuentes et al. present their results of the ultrasound (US) assessment of rectus femoris muscle in 18 chronic respiratory patients and 17 healthy controls [2]. In their study on applicability of ultrasound for muscle mass assessment in older patients with suspected sarcopenia secondary to chronic obstructive pulmonary disease, they confirmed the agreement between the results of ultrasound evaluation, namely a cross-sectional area of the rectus femoris muscle on one hand, and bioimpedance analysis (BIA) of body fatfree mass on the other. Additionally, what seems of a particular importance for geriatric research and practice, they showed a relationship between the cross-sectional area of the rectus femoris, as measured with US, and the functional measure of quadriceps muscle, that is its strength determined by a maximum voluntary isometric contraction [2].

Presented results should be discussed in light of the recent launch of the revised European consensus on sarcopenia, during the European Geriatric Medicine Society (EuGMS) Congress in Berlin. The European Working Group on Sarcopenia in Older People 2 (EWGSOP2) in its statement aimed to increase awareness of sarcopenia, and can be viewed as a call for action on prompt and accurate sarcopenia diagnosis and treatment. EWGSOP2, for the first time, included the

This comment refers to the article available at https://doi. org/10.1007/s41999-018-0130-7.

Karolina Piotrowicz

karolina.piotrowicz@uj.edu.pl

1 Department of Internal Medicine and Gerontology, Faculty of Medicine, Jagiellonian University Medical College,

Śniadeckich 10 Str., 31-351 Kraków, Poland ultrasound assessment of muscles which is listed as promising "alternative or new test and tools" [1]. Being not only absolutely safe, completely non-invasive, and radiation-free instrument, but also handy, accessible in most clinical settings, relatively inexpensive tool, US has an obvious advantage over radiological techniques employed in the diagnosis of sarcopenia thus far $[1,3]$. Of note, along the lines outlined by EWGSOP2, US assessment correlates well not only with muscle mass but also muscular function which in the new consensus has been justly given a prominent role [1]. The latter is an important methodological aspect related to the use of US in muscle assessment not to be underestimated. Clearly, when compared to the routine modalities used for this purpose, namely the bioimpedance analysis or dualenergy X-ray absorptiometry (DXA), US surpasses them by providing not only quantitative (mass) but also qualitative (correlation with muscle strength) estimates.

The great strength of the study of Ramirez-Fuentes et al. lays in its practicality and extensibility to the real-life case scenarios. With the rapid growth of the segment of population above the age of 80 years, and related increase in burden of multimorbidity and multiple functional deficits, there is a need for a quick, standardized assessment modality to assess muscle mass, architecture and function, that would be feasible in different clinical settings, including the bedside. Furthermore, as showed by Ramirez-Fuentes et al., the association between muscle quantity and quality evaluated with US, makes it possible to screen and diagnose sarcopenia also in bedridden, cognitively impaired patients who would not comply with any of the available functional assessments. Importantly, the study by Ramirez-Fuentes et al. has been designed in line with the recently published by Perkisas et al., the evidence for the protocol of standardized ultrasound assessment for sarcopenia in old age [3].

As it has been stressed by the new European consensus on sarcopenia, there are still considerable knowledge gaps, concerning the pathophysiology, diagnosis and treatment of sarcopenia [1]. In the realm of diagnostic procedures, this is 
especially true of US. The study by Ramirez-Fuentes et al. is among the very first reports concerning US applicability for skeletal muscles assessment showing the methodologic feasibility and value of the approach. It can thus be viewed as an incentive for more extensive research and institution of better quality of care and accurate reporting of sarcopenia. One of the examples of ongoing projects on the issue is the International Registry on Sarcopenia in Clinical Practice led by the Special Interest Group of the European Geriatric Medicine Society, which aims at further sarcopenia research with other aspects of ageing and morbidity in older persons [4].

\section{Compliance with ethical standards}

Conflict of interest On behalf of all authors, the corresponding author states that there is no conflict of interest.

Ethical approval This manuscript does not contain any studies with human participant performed by any of the authors.

Informed consent For this type of study, informed consent is not required.

Open Access This article is distributed under the terms of the Creative Commons Attribution 4.0 International License (http://creativeco mmons.org/licenses/by/4.0/), which permits unrestricted use, distribution, and reproduction in any medium, provided you give appropriate credit to the original author(s) and the source, provide a link to the Creative Commons license, and indicate if changes were made.

\section{References}

1. Cruz-Jentoft AJ, Bahat G, Bauer J et al (2018) Sarcopenia: revised European consensus on definition and diagnosis. Age Ageing. https://doi.org/10.1093/ageing/afy 169

2. Ramírez-Fuentes C, Mínguez-Blasco P, Ostiz F et al (2018) Ultrasound assessment of rectus femoris muscle in rehabilitation patients with chronic obstructive pulmonary disease screened for sarcopenia: correlation of muscle size with quadriceps strength and fat-free mass. Eur Geriatr Med. https://doi.org/10.1007/s4199 9-018-0130-7

3. Perkisas S, Baudry S, Bauer J et al (2018) Application of ultrasound for muscle assessment in sarcopenia: towards standardized measurements. Eur Geriatr Med. https://doi.org/10.1007/s4199 9-018-0104-9

4. Sanchez-Rodriguez D, Bruyère O (2018) The International Registry of patients with sarcopenia: applying research in sarcopenia to clinical practice. Eur Geriatr Med. https://doi.org/10.1007/s4199 9-018-0108-5 